\title{
Distance and mass of pulsating stars from multicolour photometry and atmospheric models
}

\begin{abstract}
S. Barcza ${ }^{\star}$
Konkoly Observatory, PO Box 67, 1525 Budapest, XII, Hungary

Received 15 October 2002 / Accepted 27 February 2003

Abstract. For determining distance and mass of pulsating stars a new, purely photometric method is described in which radial velocity observations are not needed. From multicolour photometry the variation of angular diameter is determined in a conventional way by using the surface brightness of the theoretical atmospheric models ATLAS of Kurucz (1997). As a function of phase the following two parameters are introduced in the Navier-Stokes equation: gravity (of the appropriate static ATLAS model), and angular diameter. Distance and mass are derived from phases of standstill. Conclusions are drawn on the hydrodynamic behaviour of the atmosphere. The new method is compared with the Baade-Wesselink (BW) method. As an example the RR Lyrae variable SU Dra is given: using $U B V R_{\mathrm{C}}$ photometry, a distance of $647 \pm 16 \mathrm{pc}$, and a mass of $0.66 \pm .03 \mathcal{M}_{\odot}$ were found. In addition to the radius change (4.58-5.51) $R_{\odot}$ radius undulations have been found with amplitude $\approx 0.2 R_{\odot}$ and period $P / 5$, which are synchronized with the main period $P=0 \mathrm{~d} 66042$. The present method has confirmed the distance value from the BW method; thus, in the problem of different distance scales from BW and other methods the short extragalactic distance scale has been bolstered by an independent argument. The mass is some 30 percent larger than the value from the equation of pulsation of van Albada \& Baker (1971).
\end{abstract}

Key words. stars: variables: RR Lyr - stars: fundamental parameters: distance, mass - stars: atmospheres stars: individual: SU Dra

\section{Introduction}

The availability of ATLAS stellar atmospheric models (Kurucz 1997) made it possible to account for the monochromatic flux of non-variable stars with a formerly unprecedented accuracy. As a function of the parameters effective temperature $T_{\mathrm{e}}$, surface gravity $g$, chemical composition, etc., large grids of models are now available for different physical assumptions on convection and turbulence. $\mathrm{Ab}$ initio knowledge or reconstruction of photometric filter functions made it possible to compute absolute fluxes in a variety of photometric bands and to fit them to stellar magnitudes and colours by an appropriate choice of zero points. The comparison of observed colours of stars with computed colours of model atmospheres made it possible to determine the physical parameters of a star. These new possibilities opened the way to explore the atmospheric parameters of variable stars which pulsate in such a manner that quasi-static approximation (QSA, Buonaura et al. 1985) is valid.

The synthetic colours $U B V R I J K$ of new and more sophisticated versions of the ATLAS models with increased microturbulence were used by Liu \& Janes $(1989,1990)$ to determine fundamental parameters of RR Lyrae stars using the BaadeWesselink (BW) technique. There are, however, many problems with the BW method: a theoretical overview of these was given by Gautschy (1987) while practical questions and the

\footnotetext{
* e-mail: barcza@konkoly.hu
}

application for RR Lyrae stars were discussed in detail by e.g. Liu \& Janes (1990), Jones et al. (1992).

The present paper will approach the determination of distance and mass of pulsating stars, especially RR Lyrae stars, from a modified point of view compared to a conventional the BW analysis. The synthetic colours and fluxes in different photometric bands of ATLAS models (Castelli 1999) will be used to determine the variation of stellar angular diameter by a similar, slightly extended method as in the BW analysis. However, the angular diameters and values of the effective gravity $g_{\mathrm{e}}$ will be introduced in the Navier-Stokes (N-S) equation averaged over the continuum-forming layers: the distance and mass of the star will be determined from the momentum balance of the variable atmosphere. In this manner the most time consuming radial velocity observations are not necessary, their problematic conversion to radius changes becomes unnecessary, and the fundamental parameters distance and mass will be obtained simultaneously by a procedure which is different from the BW technique.

Section 2 describes the method generally and its adaptation to $U B V R_{\mathrm{C}}$ photometry which is a compromise between the number of available observations and the efficiency in determining physical parameters of a pulsating stellar atmosphere. Section 3 summarizes the light and colour curves of SU Dra (=BD $\left.+67^{\circ} 652\right)$ from $U B V(R I)_{\mathrm{C}}$ observations and the results from the method as an example. In Sect. 4 the results are 
discussed and compared with those from BW analysis. The conclusions are given in Sect. 5 .

Spherical symmetry of the pulsation (excitation of radial modes) will be assumed. Cgs units will be used throughout; departures from them will be indicated. $r, \theta, \phi$ will denote spherical coordinates with their origin in the stellar centre of mass.

\section{The method}

Four steps are involved.

1. The observed magnitudes are converted to fluxes at zero air mass and corrected for interstellar extinction.

2. The observed colour indices are converted to physical quantities of the theoretical atmospheric models: i.e. to $T_{\mathrm{e}}$, $g_{\mathrm{e}}$, and surface flux in physical units for the different photometric bands including bolometric flux. In this step the bolometric flux is obtained from $T_{\mathrm{e}}$ by the StefanBoltzmann law while in the photometric bands the zero point of the magnitude scale must be determined to obtain the flux in physical units.

3. From the combination of observed and model fluxes the variable angular diameter is obtained which is introduced in the N-S equation together with the values $g_{\mathrm{e}}$.

4. At a fixed phase (time interval) the N-S equation is an algebraic equation for the unknown quantities distance and mass. It must be written out for two phases at least, from which the unknowns are obtained by elementary operations.

\subsection{Selection of the photometric system}

A photometric system with colour indices $C_{i}$ is appropriate for the present method if the physical quantities are single-valued functions $T_{\mathrm{e}}\left(C_{1}, C_{2}\right), \lg g\left(C_{1}, C_{2}\right), \ldots$ and they change slowly, i.e. the finite differences $\left|\Delta T_{\mathrm{e}} / \Delta C_{i}\right|,\left|\Delta g / \Delta C_{i}\right|$, $\mid \Delta$ surface brightness $/ \Delta C_{i} \mid$ are small for one of the indices $C_{i}$ at least. E.g. in the ranges $6000<T_{\mathrm{e}}<8000 \mathrm{~K}, 2<\lg g_{\mathrm{e}}<4$ of an RR Lyrae star for $U B V$ photometry

$\Delta T_{\mathrm{e}} /\left.\Delta(B-V)\right|_{U-B=\text { const }} \approx-10^{4} \mathrm{~K} / \mathrm{mag}$,

$\Delta \lg g_{\mathrm{e}} /\left.\Delta(U-B)\right|_{B-V=\mathrm{const}} \approx-10 \mathrm{dex} / \mathrm{mag}$

which result in errors $\approx 100 \mathrm{~K}, 0.1 \mathrm{dex}$ for a typical error $0.01 \mathrm{mag}$ in the colour index. For $B V R_{\mathrm{C}}$ photometry

$\Delta \lg g_{\mathrm{e}} /\left.\Delta(B-V)\right|_{V-R_{\mathrm{C}}=\text { const }} \approx 70 \mathrm{dex} / \mathrm{mag}$,

$\Delta \lg g_{\mathrm{e}} /\left.\Delta\left(V-R_{\mathrm{C}}\right)\right|_{B-V=\text { const }} \approx-70 \mathrm{dex} / \mathrm{mag}$

which make this photometry unfit to determine $g_{\mathrm{e}}$. The final result is that $T_{\mathrm{e}}, \lg g_{\mathrm{e}}$ and the flux $\mathcal{F}$ in different photometric bands are found with reasonable accuracy from a pair of the colour indices. The conversion $C_{i} \rightarrow T_{\mathrm{e}}$ etc. can be made conveniently using the Kurucz tables (1997) in which the interstellar reddening is included.

\subsection{The photometric half angular diameter}

We list some useful relations for convenience. At a phase $\varphi$ in the photometric band $x$ the luminosity of the star is

$L_{x}(\varphi)=4 \pi R_{0}^{2}(\varphi) \mathcal{F}_{x}(\varphi)$

where the radius $R_{0}$ belongs to zero optical depth in the reference frame with origin in the stellar centre of mass,

$\mathcal{F}_{x}=\int_{0}^{\infty} \mathrm{d} \lambda S_{x}(\lambda) \int_{0}^{\infty} \mathrm{d} \tau_{\lambda} \sum_{i=1}^{6} c_{i} B_{\lambda}\left(\tau_{\lambda_{i}}\right)$

is the physical flux (Kurucz 1979) on the stellar surface in the photometric band $x$ where $S_{x}(\lambda)$ is the filter function defining the colour system. The monochromatic flux on the stellar surface was approximated in Eq. (4) by a sum, where $c_{1}, \ldots, c_{6}$ and $\tau_{\lambda_{1}}, \ldots, \tau_{\lambda_{6}}$ are $0.1615,0.1346,0.2973,0.1872,0.1906$, 0.0288 , and $0.038,0.154,0.335,0.793,1.476,3.89$ respectively (Traving et al. 1966), $B_{\lambda}$ is the Planck function which accounts well for the source function if the stellar continuum is considered. The meaning of Eq. (4) is that the monochromatic flux originates roughly from six layers between $R_{\tau_{\lambda} \approx 3.89} \leq r \leq$ $R_{\tau_{\tau} \approx 0.038}$ and the main contribution comes from the neighbourhood of $R_{\tau_{\imath} \approx 0.335}$. These (wavelength dependent) radii are defined e.g. by

$\tau_{\lambda}=0.335=-\int_{R_{0}}^{R_{\tau_{\lambda}=0.335}} \kappa_{\lambda}(r) \mathrm{d} r$,

$\kappa_{\lambda}$ is the monochromatic absorption coefficient. If $\kappa_{\lambda}(r)$ is a smooth and moderately changing function of $\lambda$ we can assume that $R_{1}=\overline{R_{\tau_{\lambda}=0.038}}, R=\overline{R_{\tau_{\lambda}=0.335}}, R_{2}=\overline{R_{\tau_{\lambda}=3.89}}$ are independent of wavelength (overlining denotes averaging over $\lambda$ ). If $x$ represents bolometric or an optical band the Rosseland optical depth can be substituted for $\tau_{\lambda}$ to estimate $R_{1}, R, R_{2}$, which are now automatically independent of the wavelength. $R_{0}$ is the top of the atmosphere, $R_{1}, R_{2}$ can be regarded as the top and bottom of the continuum-forming atmospheric region. $\left(R_{1}-R_{2}\right) / R_{0}$, $R / R_{0}$ vary with $\varphi, R_{2}<R<R_{1}$. These variations reflect the change of $\lg g_{\mathrm{e}}(\varphi)$.

At distance $d$ the observed stellar flux $\mathcal{I}_{x}$ will be

$\mathcal{I}_{x}(\varphi)=10^{-A_{x} / 2.5} L_{x}(\varphi) / 4 \pi d^{2}$

where $A_{x}$ is the interstellar extinction in magnitudes. The half angular diameter of the star is obtained for zero optical depth by

$\vartheta_{0}(\varphi)=R_{0}(\varphi) / d=\left[10^{A_{x} / 2.5} \mathcal{I}_{x}(\varphi) / \mathcal{F}_{x}(\varphi)\right]^{1 / 2}$.

$\mathcal{I}_{x}$ must be derived from observed magnitudes and compared with the tabulated value $\mathcal{F}_{x}$ of the theoretical models which are found by a comparison of observed colour indices with those of computed models.

The conventional way to determine $\vartheta_{0}(\varphi)$ is to introduce $A_{x}$, $\mathcal{I}_{x}(\varphi)=S_{0} 10^{-\left[m_{x}(\varphi)+\mathrm{BC}_{x}(\varphi)\right] / 2.5}$, and $\mathcal{F}_{\text {bol }}(\varphi)=a T_{\mathrm{e}}^{4}(\varphi)$ in Eq. $(7)$ where $a$ is the Stefan-Boltzmann constant and the zero points of the magnitude scales $m_{x}, \mathrm{BC}_{x}$ are merged in the constant $S_{0}$ (e.g. Liu \& Janes 1990, $x=V$ ), i.e. two parameters of theoretical models $\left(\mathrm{BC}_{V}, T_{\mathrm{e}}\right)$ belonging to a pair of observed colour 
indices are needed. This procedure was applied in the present paper as well; the actual form was

$\vartheta_{0}=0.636 \times 10^{-\left(V+\mathrm{BC}_{V}-2 A_{V}\right) / 5} T_{\mathrm{e}}^{-2}$

in radians. To check Eq. (8) a single parameter determination of $\vartheta_{0}$ was used by the following formulae in Eq. (7). An apparent magnitude $X$ is equivalent to a physical flux

$\mathcal{I}_{X}=10^{-7+\left(X_{\mathrm{Vega}}-X\right) / 2.5} \iota_{X}^{\text {(Vega) }} \operatorname{ergs~s}^{-1} \mathrm{~cm}^{-2}$,

where $X_{\text {Vega }}=0.03,0.039 \mathrm{mag}, \iota_{X}^{(\text {Vega })}=1.82,1.04$ if $X=$ $V, R_{\mathrm{C}}$, respectively. In the band $X$ the physical flux of a model is

$\mathcal{F}_{X}=10^{-\left(X_{\mathrm{K}}-4.495\right) / 2.5} \mathrm{ergs} \mathrm{s}^{-1} \mathrm{~cm}^{-2}$

where $X_{\mathrm{K}}$ is the tabulated magnitude of the Kurucz (1997) tables for $U, B, V, R_{\mathrm{C}}, I_{\mathrm{C}}$. The derivation of Eqs. (9) and (10) is given in Appendix A.

\subsection{The momentum balance of a radially pulsating atmosphere}

For a non-rotating star the $r$ component of the N-S equation is

$$
\begin{array}{r}
\frac{\partial v_{r}(r, \theta, \phi, t)}{\partial t}+v_{r} \frac{\partial v_{r}}{\partial r}+a_{1}(r, \theta, \phi, t)=-\frac{G \mathcal{M}}{r^{2}} \\
-\frac{1}{\rho(r, \theta, \phi, t)} \frac{\partial p(r, \theta, \phi, t)}{\partial r}+a_{2}(r, \theta, \phi, t)
\end{array}
$$

(Landau \& Lifshitz 1980) where $v_{r}$ is the $r$ component of the velocity, $G$ is the gravitational constant, $\mathcal{M}$ is the stellar mass, $\rho$ is the density. $a_{1}=\left[v_{\theta}(r, \theta, \phi, t) \partial v_{r} / \partial \theta+\right.$ $\left.\sin ^{-1} \theta v_{\phi}(r, \theta, \phi, t) \partial v_{r} / \partial \phi-v_{\theta}^{2}(r, \theta, \phi, t)-v_{\phi}^{2}(r, \theta, \phi, t)\right] / r$ is the rest of the terms from $(\boldsymbol{v}, \boldsymbol{\nabla}) v_{r}$. The sum of the terms from the molecular viscosity is $a_{2}$. Setting the sound velocity (about $10^{6} \mathrm{~cm} / \mathrm{s}$ ) for the components $v_{\theta}, v_{\phi}$ as an upper limit, the order of magnitude of $a_{1}$ will be $10 \mathrm{~cm} / \mathrm{s}^{2}$; therefore, except for phases of stationary flow (i.e. $\partial v_{r} / \partial t \approx 0$ ) this term is negligible in comparison with the explicitly written terms. Estimations are not available for $a_{2}$, however, it can be assumed to be negligible in comparison with the other terms, especially at phases of standstill (i.e. $v_{r} \approx 0$ ).

For a static stratification the acceleration

$g_{\mathrm{e}}=-\frac{1}{\rho} \frac{\partial p}{\partial r}$

is constant within few percents in the domain $R_{2}<r<R_{1}$. On the right hand side of Eq. (11) the second term can be approximated by a constant $g_{\mathrm{e}}$ which is obtained from the colourcolour diagram. To take all dynamical corrections into account the term $a_{d}(r, \theta, \phi, t)=a_{1}+v_{r} \partial v_{r} / \partial r+\cdots$ will be introduced; “..." represents the terms which were not listed here, e.g. a dynamical correction in Eq. (12). However, the spatial average $g_{\mathrm{d}}$ of $a_{d}$ is expected to be small in the phases of standstill in comparison to the acceleration $\partial v_{r} / \partial t$.

Perfect spherical symmetry of the velocity field will be assumed; the terms describing eventual non-radial modes are assumed to be zero:

$v_{r}(r, t)=v_{r}^{(0)}(t)+\left.\frac{\partial v_{r}}{\partial r}\right|_{r=R}(R-r)+\cdots=\dot{R}(t)+\cdots$, the homogeneous velocity $\dot{R}$ is a good first approximation, the next term would account for the compression or expansion of the atmosphere, i.e. for a change of $R_{1}-R_{2}$. By introducing Eqs. (12), (13) in Eq. (11) and averaging over the interval $R_{2} \leq$ $r \leq R_{1}$ (i.e. multiplying by $r^{2} \sin \theta$, dividing by $4 \pi\left(R_{1}^{3}-R_{2}^{3}\right) / 3$, integrating over $r, \theta, \phi)$ the equation

$\frac{\partial \bar{v}_{r}(R, t)}{\partial t}=-\frac{G \mathcal{M}}{R^{2}(t)}+g_{\mathrm{e}}(t)+g_{\mathrm{d}}(R, t)$

is obtained.

Now all terms are neglected in Eq. (13) except for $\dot{R}$ and an apparent angular change $\vartheta_{\mathrm{a}}=R_{\mathrm{a}} / d$ is introduced such that

$R_{0}(t)=R(t)+R_{\mathrm{a}}(t)=\left[\vartheta(t)+\vartheta_{\mathrm{a}}(t)\right] d$,

$R_{\mathrm{a}} \ll R$ accounts for the angular change by opacity as a consequence of the variation of $g_{\mathrm{e}}$. In a turning point of $\vartheta_{0}$, i.e. at standstill of the atmosphere, $\dot{\vartheta}_{0} \approx 0, \dot{\vartheta}_{\mathrm{a}} \approx 0,\left|\partial v_{r} / \partial t\right| \gg$ $\left|v_{r} \partial v_{r} / \partial r\right|$, thus, Eq. (14) takes the form

$\left[\ddot{\vartheta}_{0}(t)-\ddot{\vartheta}_{\mathrm{a}}(t)\right] d=-G \frac{\mathcal{M}}{\vartheta^{2}(t) d^{2}}+g_{\mathrm{e}}(t)+g_{\mathrm{d}}(t)$

and $g_{\mathrm{d}}$ is expected to be small.

The functions $\vartheta_{0}(t)$ and $g_{\mathrm{e}}(t)$ can be determined from the observed colour indices and brightness, $\ddot{\vartheta}_{0}$ can be obtained by numerical differentiation. $R_{\mathrm{a}}$ can be estimated by interpolation from the Kurucz tables (1979); compared to $R_{0}$ it is negligible if $\lg g_{\mathrm{e}}>2.5$. $\ddot{R}_{\mathrm{a}}$ can be estimated by numerical differentiation; it is negligible during the pulsation except for the phases in which $g_{\mathrm{e}}$ changes strongly. From continuity of $\vartheta_{0}(t)$ it follows that there exist two turning points at least: $t_{1}, t_{2}$. Writing Eq. (16) for $t=t_{1}$ and $t_{2}$, neglecting $g_{\mathrm{d}}$, and solving the two equations leads to:

$d=\frac{g_{\mathrm{e}}\left(t_{1}\right)+\ddot{R}_{\mathrm{a}}\left(t_{1}\right)-\left[g_{\mathrm{e}}\left(t_{2}\right)+\ddot{R}_{\mathrm{a}}\left(t_{2}\right)\right]\left[\vartheta\left(t_{2}\right) / \vartheta\left(t_{1}\right)\right]^{2}}{\ddot{\vartheta}_{0}\left(t_{1}\right)-\ddot{\vartheta}_{0}\left(t_{2}\right)\left[\vartheta\left(t_{2}\right) / \vartheta\left(t_{1}\right)\right]^{2}}$,

$\mathcal{M}=\left[g_{\mathrm{e}}(t)-\ddot{\vartheta}(t) d\right] \vartheta^{2}(t) d^{2} / G$

Since $\vartheta_{\mathrm{a}}$ is a small correction to $\vartheta_{0}$ Eqs. (17) and (18) were solved while neglecting the apparent change of radius $\left(R_{\mathrm{a}}=0\right.$, $\ddot{R}_{\mathrm{a}}=0$, i.e. $\left.\vartheta \equiv \vartheta_{0}\right)$ and taking into account $R_{\mathrm{a}} \neq 0, \ddot{R}_{\mathrm{a}} \neq 0$, $\vartheta_{\mathrm{a}}=R_{\mathrm{a}} / d$.

\section{Distance and mass of SU Dra, some physical parameters of its atmosphere}

As an example some parameters of the RR Lyrae variable SU Dra will be determined. This star has a stable light curve without Blazhko effect; however, the analysis of the photometric observations left some ambiguity whether secondary variations of some 10 mmag do exist (Barcza 2002, Paper I). SU Dra is a metal-deficient Population II star: $[M]=-1.6$ dex. Its galactic coordinates are $l=133.45, b=+48.27$ i.e. it lies high above the disk, the interstellar reddening is small in that direction: $E(B-V)=0.015 \mathrm{mag}$ (Liu \& Janes 1990). Its photometric behaviour was described in Paper I; from this observational material the segments $k=25-51$ were used to 
Table 1. $U B V(R I)_{\mathrm{C}}$ magnitudes of SU Dra on HJD $=2452054$, segment $k=52$. The fraction of HJD is given for $V$, the epochs of $B$, $U, R_{\mathrm{C}}, I_{\mathrm{C}}$ are obtained by adding $-.0007,-.0018, .0005, .0010$ to the epoch of $V$.

\begin{tabular}{rrrrrrr}
\hline \hline HJD & $\varphi$ & $V$ & $B$ & $U$ & $R_{\mathrm{C}}$ & $I_{\mathrm{C}}$ \\
\hline .3906 & .114 & 9.492 & 9.683 & 9.717 & 9.322 & 9.087 \\
.4072 & .139 & 9.546 & 9.757 & 9.770 & 9.363 & 9.105 \\
.4111 & .145 & 9.559 & 9.773 & 9.789 & 9.367 & 9.111 \\
.4150 & .151 & 9.573 & 9.793 & 9.806 & 9.381 & 9.119 \\
.4314 & .176 & 9.614 & 9.853 & 9.861 & 9.402 & 9.136 \\
.4353 & .182 & 9.624 & 9.870 & 9.875 & 9.432 & 9.159 \\
.4391 & .187 & 9.636 & 9.881 & 9.885 & 9.416 & 9.146 \\
.4553 & .212 & 9.676 & 9.950 & 9.960 & 9.466 & 9.182 \\
.4592 & .218 & 9.686 & 9.965 & 9.968 & 9.458 & 9.188 \\
.4630 & .224 & 9.694 & 9.980 & 9.980 & 9.473 & 9.175 \\
.4791 & .248 & 9.737 & 10.045 & 10.046 & 9.492 & 9.202 \\
.4830 & .254 & 9.750 & 10.058 & 10.052 & 9.503 & 9.210 \\
.4868 & .260 & 9.762 & 10.076 & 10.063 & 9.500 & 9.203 \\
.5062 & .289 & 9.797 & 10.122 & 10.109 & 9.528 & 9.232 \\
.5110 & .296 & 9.807 & 10.146 & 10.136 & 9.546 & 9.240 \\
.5148 & .302 & 9.817 & 10.149 & 10.147 & 9.551 & 9.245 \\
.5311 & .327 & 9.845 & 10.188 & 10.178 & 9.557 & 9.254 \\
.5349 & .332 & 9.848 & 10.203 & 10.199 & 9.567 & 9.258 \\
.5388 & .338 & 9.854 & 10.210 & 10.207 & 9.576 & 9.258 \\
\hline
\end{tabular}

compile an average $U B V(R I)_{\mathrm{C}}$ light curve because these observations were performed using two comparison stars. To improve the phase coverage a new segment $k=52$ was added containing $19 U B V(R I)_{\mathrm{C}}$ observations on JD $=2452054$ with the Wright camera attached to the $1 \mathrm{~m}$ Ritchey-Chretien telescope of the Konkoly Observatory, Table 1 reports these results. Technical details of the observations and reduction were the same as in segments $k=42-51$ (Paper I).

\subsection{Observed UBV $(R I)_{C}$ light and colour curves}

The observed magnitudes $U, B, R_{\mathrm{C}}$ were linearly interpolated to the epoch of the $V$ observations to avoid systematic errors of colour indices $U-B, B-V, V-R_{\mathrm{C}}$ in the rising branch. A comparison with the colour indices of Liu \& Janes (1989) indicated small systematic differences between the colour systems at Konkoly Observatory and Kitt Peak. Since it is of primary importance to have the same filter functions in the observations and computation of synthetic colours of the atmospheric models, the colours of the Konkoly observations have been shifted by $\Delta(B-V)=+0.029, \Delta(U-B)=+0.026$, $\Delta\left(V-R_{\mathrm{C}}\right)=+0.019 \mathrm{mag}$. By these shifts the brightness $V$ and the colour indices of one of the comparison stars, BD $+67^{\circ} 708$, became identical in the Konkoly and Kitt Peak colour systems as is obvious from Table 3 of Paper I, and the systematic differences of the Konkoly and Kitt Peak curves disappeared. Figure 1 is a plot of the observed magnitudes $V$ and the colour
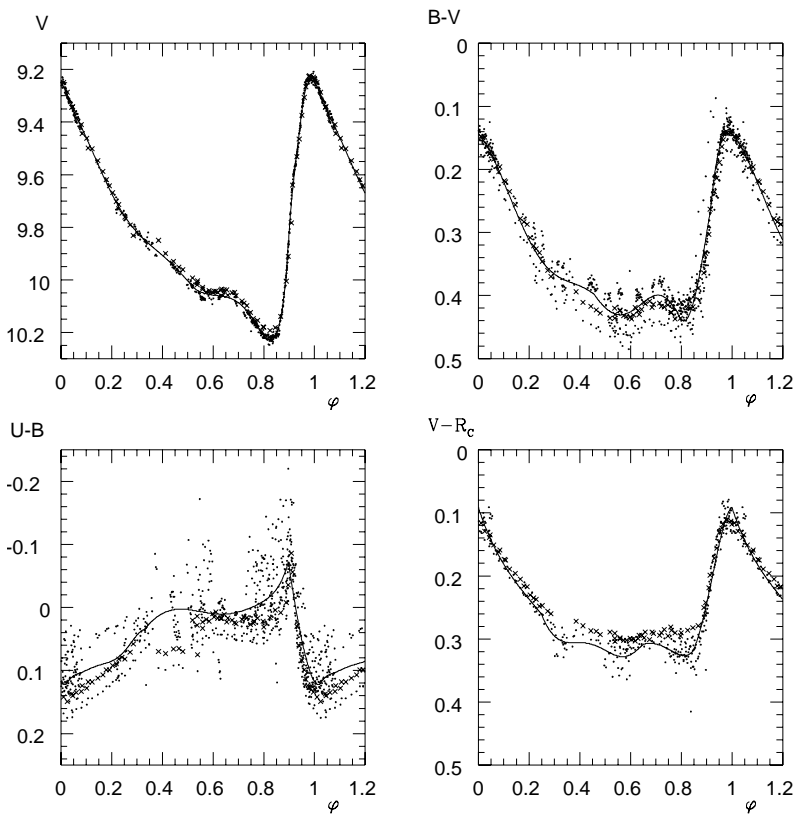

Fig. 1. Light curve $V$ and colour curves of SU Dra. Dots: segments $k=25-29,42-51$ from Paper I and $k=52$, all are shifted by the amount indicated in the text. Crosses: segments $k=30-41$ from Liu $\&$ Janes (1989). Lines: fitted curves to segments $k=25-52$.

indices; the phase was computed according to Paper I, taking into account the secular change of period and phase noise. The curves drawn in Fig. 1 were obtained by high-order spline functions, they were fitted to the shifted colour curves. These curves are the basis for deriving $T_{\mathrm{e}}(\varphi), \lg g_{\mathrm{e}}(\varphi), \mathcal{F}_{X}(\varphi), \mathcal{I}_{X}(\varphi)$ with $X=V, R_{\mathrm{C}}, \mathrm{BC}_{V}$, and $\vartheta_{0}(\varphi)$ according to Sect. 2 .

The colour curves $V-I_{\mathrm{C}}$ were determined as well; however, they were omitted from the analysis because the CCD realization of $I_{\mathrm{C}}$ was not free of systematic errors; these originated from the red cut-off which is considerably different from that of a GaAs cathode and of the improved $S_{I_{\mathrm{C}}}(\lambda)$ of Bessell (1990).

The fitted curves show definite structure around $\varphi \approx 0.4,0.7$ in addition to the ascending branch which is their main feature. If only 60-70 points are observed (e.g. Liu \& Janes 1989) the fine structure of the light and colour curves remains hidden. It could be revealed by the 674 observed points in the present study.

\subsection{Effective temperature, gravity, angular diameter}

The synthetic colours from the Kurucz (1997) tables (Castelli 1999) were interpolated linearly to $[M]=-1.6$ dex and $E(B-$ $V)=0.015 . T_{\mathrm{e}}(\varphi), \lg g_{\mathrm{e}}(\varphi)$, tabular magnitudes $V, R_{\mathrm{C}}, \mathrm{BC}_{V}$ were interpolated to a pair of colour indices $(U-B, B-V),(U-$ $\left.B, V-R_{\mathrm{C}}\right),\left(U-V, V-R_{\mathrm{C}}\right) . V, R_{\mathrm{C}}$ were converted to surface flux $\mathcal{F}_{V}, \mathcal{F}_{R_{\mathrm{C}}}$ by Eq. (10).

Panel (a) of Fig. 2 shows the theoretical isogravity curves $\lg g(U-B, B-V)=2,3,4$ for different values of $T_{\mathrm{e}}$, the isotherm curves $T_{\mathrm{e}}(U-B, B-V)=6,7,8 \times 10^{3} \mathrm{~K}$ for different values of $\lg g$, and the observed colour loop $(U-B, B-V)$ as a function of $\varphi$. Panels (b)-(d) are a plot of $\lg g_{\mathrm{e}}(\varphi), \vartheta_{0}(\varphi)$, $T_{\mathrm{e}}(\varphi)$ for the colour loop. $\vartheta_{0}(\varphi)$ was determined from Eq. (8) 

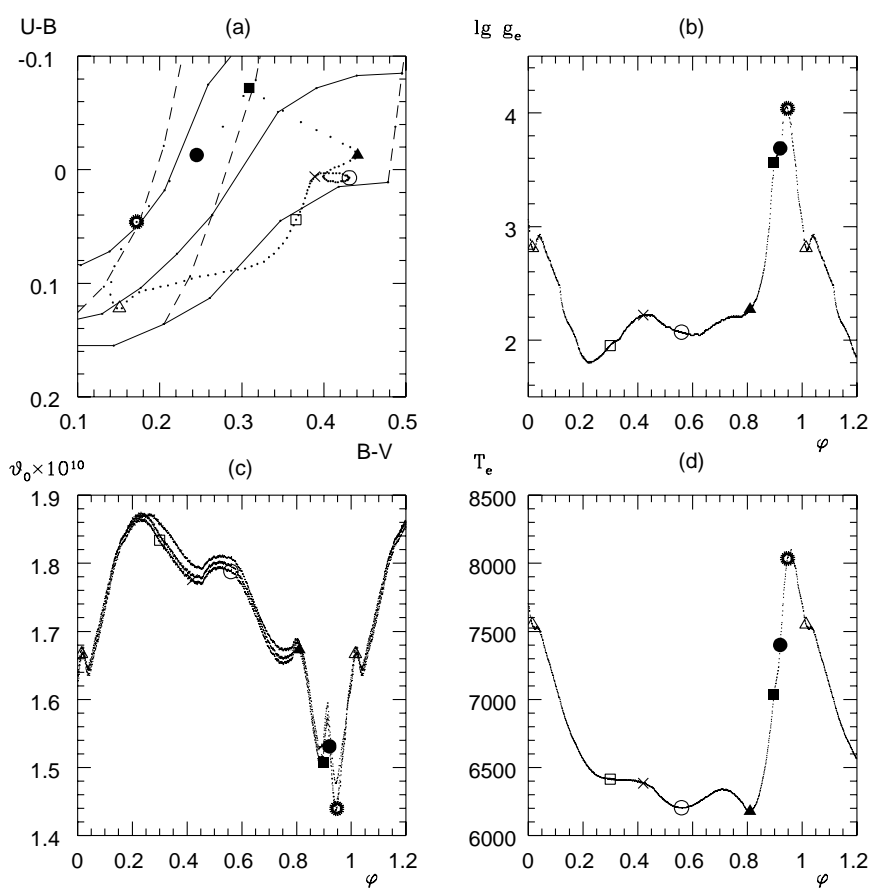

Fig. 2. Panel a): lines: from bottom to top the isogravity curves $\lg g=2,3,4$, dashed lines: from left to right the isotherm curves $T_{\mathrm{e}}=$ $8000,7000,6000 \mathrm{~K}$. The curves are interpolated to $[\mathrm{M}]=-1.6, E(B-$ $V)=0.015$. Dots: $U-B, B-V$ loop of SU Dra. Characteristic points of the $\operatorname{loop} \varphi=0.015$ and $1.015,0.3,0.42,0.56,0.81,0.897,0.92,0.947$ are marked by different symbols which are plotted in Panels b)-d) as well. Panels b), d): the interpolated $\lg g_{\mathrm{e}}, T_{\mathrm{e}}$ values. Panel c): $\vartheta_{0}$ from Eqs. (7), (9), and (10) with $V, R_{\mathrm{C}}$ and from Eq. (8). The symbols of the loop indicate $\vartheta_{0}(\varphi)$ from Eq. (8).

and from $\left(\mathcal{I}_{V}, \mathcal{F}_{V}\right),\left(\mathcal{I}_{R_{\mathrm{C}}}, \mathcal{F}_{R_{\mathrm{C}}}\right)$ by Eqs. (7), (9) and (10): it is remarkable that the curves are congruent within 2 percent. This finding demonstrates that in the range $\lg g_{\mathrm{e}}, T_{\mathrm{e}}$ of an RR Lyrae star the simultaneous use of $B-V$ and $U-B$ estimates $T_{\mathrm{e}}$ well. Therefore, the infrared photometry can be dropped; it is more complicated from an observational point of view and was expected to give $T_{\mathrm{e}}$ correctly by observing the slope of the continuum over a larger wavelength interval (Liu \& Janes 1990).

The procedure was performed with the colour-colour curves $\left(U-B, V-R_{\mathrm{C}}\right),\left(U-V, V-R_{\mathrm{C}}\right)$. The congruence of the curves from Eqs. (8) and (7), (9), (10) was within 2 percent confirming that $T_{\mathrm{e}}$ can be determined well by simultaneous use of $U-B$ or $U-V$ and $V-R_{\mathrm{C}}$. The curves $\lg g_{\mathrm{e}}(\varphi)$ etc. were of similar form with those from $(U-B, B-V)$, the differences did not exceed $0.1 \mathrm{dex}, 100 \mathrm{~K}$ in the descending branch while in the ascending branch upper limits of $0.4 \mathrm{dex}, 200 \mathrm{~K}$ were found for the differences. The curves $\vartheta_{0}(\varphi)$ were coincident to within 10 percent. The differences were attributed to non-perfect filter functions, and to the fitting procedure when the averaged colour curves were constructed. Among $\vartheta_{0}(\varphi)$ from $(U-B, B-V)$ and $\left(U-B, V-R_{\mathrm{C}}\right),\left(U-V, V-R_{\mathrm{C}}\right)$ the largest differences were in the phase interval $0.87<\varphi<1$; however, the cause for this seems to be that the curves based on $B-V$ and $V-R_{\mathrm{C}}$ respectively are asynchronous. The differences could have been removed by a phase shift; nevertheless, averaging seemed more appropriate. Therefore, at the points $\varphi$ the values $\lg g_{\mathrm{e}}(\varphi), T_{\mathrm{e}}(\varphi), \vartheta_{0}(\varphi)$ were averaged from the three combinations of colour-colour indices of $U B V R_{\mathrm{C}}$. The result is plotted in Fig. 3.

The values of $\lg g_{\mathrm{e}}(\varphi), T_{\mathrm{e}}(\varphi)$ agree well with those Siegel (1982) and Liu \& Janes (1990) determined from Strömgren and $U B V R I J K$ photometry respectively. The averaged values are $\overline{\lg g_{\mathrm{e}}(\varphi)}=2.41$ and $\overline{T_{\mathrm{e}}(\varphi)}=6490 \mathrm{~K}$ (this study) while 2.69, $6400 \mathrm{~K}$, and 2.72, $6433 \mathrm{~K}$ are given by Siegel (1982) and Liu \& Janes (1990) respectively.

The agreement between the present $\vartheta_{0}(\varphi)$ curves and those of Liu \& Janes (1990) is satisfactory. The main difference lies in the undulation of the present curve amounting to some 5 percent which was provided by ten times more photometric observations. The wavy structure of $\vartheta_{0}(\varphi)$ is remarkable: at about $\varphi \approx 0.45,0.7$ local minima of $\vartheta_{0}(\varphi)$ are clearly visible. This suggests that a sub-oscillation exists which is synchronized with the main pulsation. In the phase intervals $0.8<\varphi<0.9,0.1<\varphi<0.4$ the change of $\vartheta_{\text {a }}$ and eventual dynamical effects from the strong shock hamper us in getting a clear picture of the sub-oscillation; however, a periodic undulation with $\approx P / 5$ is clearly visible in $\vartheta_{0}(\varphi)$ and $V(\varphi)$ where $P=5.71 \times 10^{4} \mathrm{~s}$ is the period of SU Dra. To check the reality of the undulations, the $674 \mathrm{~V}$ observations were analysed by the string length minimization described in Paper I since they can be regarded as the most reliable because of the use of two comparison stars. The period $P / 5$ has been found only with a very shallow minimum of the string length.

The averaged curve $\vartheta_{0}(\varphi)$ was differentiated by midpoint formulae with a phase span of $\Delta \varphi=0.02(\approx 20 \mathrm{~min})$ which was found to be an appropriate compromise between the noise and the accuracy of $\ddot{\vartheta}_{0}$. This choice is in agreement with the present simplified hydrodynamic model: a description of more rapid structures cannot be expected. The angular acceleration $\ddot{\vartheta}_{0}$ is plotted in the lower left panel of Fig. 3.

To estimate the apparent radius change $R_{\mathrm{a}}(\varphi)$ the geometrical depth of $\tau_{\text {Ross }}=0.335$ was interpolated to $\lg g_{\mathrm{e}}(\varphi), T_{\mathrm{e}}(\varphi)$ from the Kurucz (1979) tables. $R_{0}(\varphi)=1.99 \times 10^{16} \vartheta_{0}(\varphi) \mathrm{km}$, $R(\varphi)$, and $R_{1}(\varphi)-R_{2}(\varphi)$ as error bars are plotted in Fig. 4 a. From the point of view of apparent diameter change and acceleration the critical phase intervals are $0.8<\varphi<0.9$, $0.1<\varphi<0.4$. The break of the slope of $\vartheta_{0}(\varphi)$ at $\varphi \approx 0.25$ can be interpreted by the peak of $R_{\mathrm{a}}$ which is superimposed on the true atmospheric motion $R_{0}(\varphi)$. $\ddot{R}_{\mathrm{a}}$ was obtained from differentiating polynomial fits to $R_{\mathrm{a}}$. Three points are noteworthy:

* The secondary peak of $\vartheta_{0}$ at $\varphi \approx 0.55$ coincides just with the fall of the infrared brightness $K$ which begins at phase delay $\approx 0.6$ from the visual maximum (Liu \& Janes 1989).

* Double standstill exists at minimal radius with differences $\Delta \varphi=0.031, \Delta \vartheta_{0}=\vartheta_{0}(0.947)-\vartheta_{0}(0.978) \approx 10^{-12}$ (i.e. $\left.\Delta R_{0} \approx 2 \times 10^{4} \mathrm{~km}\right)$.

* The most prominent features of $\ddot{\vartheta}_{0}$ are produced by a strong variation of $R_{\mathrm{a}}=R_{0}-R$.

\subsection{Distance and mass}

At both turning phases the fine structure of $\vartheta_{0}(\varphi)$ shows two standstills with roughly equal $\vartheta_{0}$, the averaged values of the quantities for Eqs. (17) and (18) are listed in Table 2. Selection criteria were for these phases that the atmosphere must be in 

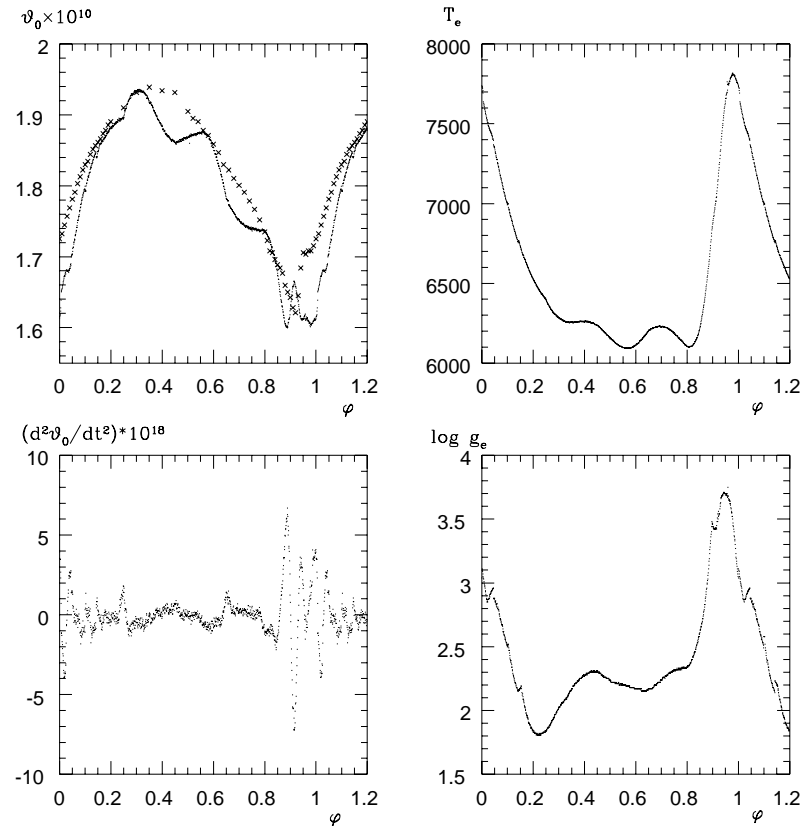

Fig. 3. Dots: averaged curves $\vartheta_{0}(\varphi), T_{\mathrm{e}}(\varphi), \lg g_{\mathrm{e}}(\varphi)$ from the loops $(U-B, B-V),\left(U-B, V-R_{\mathrm{C}}\right),\left(U-V, V-R_{\mathrm{C}}\right) . \ddot{\vartheta}_{0}$ was derived from the averaged $\vartheta_{0}(\varphi)$ by numerical differentiation. Crosses: variation of $\vartheta_{0}(\varphi)$ given by Liu \& Janes (1990) from infrared photometry using a formula of type Eq. (8).

standstill to have the smallest $g_{\mathrm{d}}$ in Eq. (14), the effective gravity must be constant to avoid strong variation of $R_{\mathrm{a}}$, and the interval of the averaging must be long enough to smooth out scatter and eventual rapid variations for which the present hydrodynamic model is oversimplified. The errors of $\lg g_{\mathrm{e}}$ and $\vartheta_{0}$ are less than 3 percent; the errors of $\ddot{\vartheta}_{0}$ and $\ddot{R}_{\mathrm{a}}$ dominate the error of $d, \mathcal{M}$. In the phase intervals $\mathrm{A}$ and $\mathrm{B}$ the atmosphere is falling freely, in C and D it is in the state of maximal compression by the shock(s?) from the deeper layers.

In the intervals A, B, C, D it can be assumed that the terms of $g_{\mathrm{d}}$ with a factor $v$ are negligible, i.e. $g_{\mathrm{e}}$ of the static atmosphere accounts well for the outward acceleration via Eq. (14). The values $g_{\mathrm{e}}$ etc. of the pairs AC, AD, BC, BD, CD were introduced in Eqs. (17) and (18), $d$ and $\mathcal{M}$ are listed in Table 3, Cols. 2-5 refer to $R_{\mathrm{a}}=0, \ddot{R}_{\mathrm{a}}=0$ and $R_{\mathrm{a}}, \ddot{R}_{\mathrm{a}}$ from Table 2, respectively. The pair AB was omitted because the errors in $g_{\mathrm{e}}$, $\ddot{\vartheta}_{0}, \ddot{R}_{\mathrm{a}}$ made $d$ and $\mathcal{M}$ unrealistic. After averaging Cols. 2 and 3 $d=(564 \pm 31) \mathrm{pc}, \mathcal{M}=(0.51 \pm .06) \mathcal{M}_{\odot}$ were found as a first approximation; the errors represent 68 percent confidence level.

Columns 4 and 5 are more surprising: they suggest

$d=(647 \pm 16) \mathrm{pc}$

$\mathcal{M}=(0.66 \pm .03) \mathcal{M}_{\odot}$.

The values using A were excluded from these averaged $d, \mathcal{M}$ on the following arguments. The atmosphere is very extended in the interval $0.15<\varphi<0.4$ i.e. its optical scale height increases to $R_{0}-R_{2} \approx 3 \times 10^{5} \mathrm{~km} \approx 0.1 R_{0}$ at $\varphi=0.225$, plane parallel approximation (used in ATLAS models for radiative transfer) is questionable (Baschek et al. 1992). The apparent acceleration exceeds the effective gravity $\left(\ddot{R}_{\mathrm{a}} \approx 2 g_{\mathrm{e}}\right), g_{\mathrm{d}} \approx 0$ is presumably not satisfied, and it begins just a strong ap-
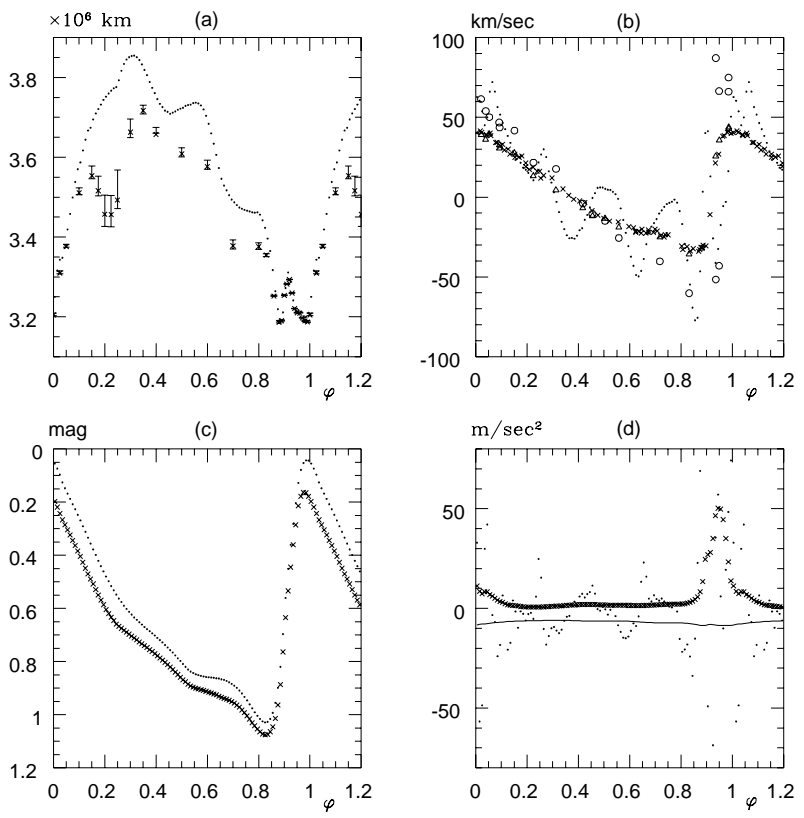

Fig. 4. Physical quantities characterizing the pulsation of SU Dra, $d=$ $1.99 \times 10^{16} \mathrm{~km}$ was used to convert the angular quantities. Panel a): dots: $R_{0}(\varphi)$, crosses: $R(\varphi)$ which belong to $\tau=0$ and $\tau_{\text {Ross }}=0.335$ respectively. The error bars indicate $R_{1}(\varphi)-R_{2}(\varphi)$. Panel b): dots: $\dot{\vartheta}_{0} d$, crosses: from CORAVEL radial velocities (Liu \& Janes 1989), triangles and circles: from radial velocities of metallic lines and $\mathrm{H}_{\gamma}$ (Oke et al. 1962) 1.32(- $\left.-v_{\text {radial }}(\varphi)-166.9\right) \mathrm{km} \mathrm{s}^{-1}$ is plotted. Panel c): dots: $V-9.2$ crosses: $M_{\text {bol }}=-2.5 \lg \left[L(\varphi) / L_{\odot}\right]+4.75$. Panel d): dots: $\ddot{\vartheta}_{0} d$, line: $-G \mathcal{M} / R^{2}(\varphi)$, crosses: $g_{\mathrm{e}}$.

parent (linear rather than accelerating?) contraction; furthermore, $\ddot{R}_{\mathrm{a}}$ can be estimated with large uncertainty. In Table 2 $\ddot{R}_{\mathrm{a}}(\varphi=0.31)=2.27 \mathrm{~m} / \mathrm{s}^{2}$ is probably an overestimation, e.g. an ad hoc correction to $\ddot{R}_{\mathrm{a}}(\varphi=0.31)=0.10 \mathrm{~m} / \mathrm{s}^{2}$ would result in $d=587,559 \mathrm{pc}$ and $\mathcal{M}=0.81,0.70 \mathcal{M}_{\odot}$ which are somewhat closer to the values from combining B, C, D. Therefore, the data from AC, AD have a very low weight; however, to give a formal standard error to the unequivocal $d$ and $\mathcal{M}$ from $\mathrm{BC}, \mathrm{BD}, \mathrm{CD}$ they were used but omitted in the averaging.

\subsection{Luminosity, radius, velocity, acceleration}

The distance modulus is

$m-M=(9.05 \pm .05) \mathrm{mag}$.

Using $R_{0}(\varphi)$ and $T_{\mathrm{e}}(\varphi)$ the intensity averaged luminosity is $\langle L\rangle=(1.68 \pm .08) \times 10^{35} \mathrm{ergs} / \mathrm{s}=(42.4 \pm 2.1) L_{\odot} ;$ the errors were derived from the error of $d$. The mean radius is $\overline{R_{0}}=3.54 \times 10^{6} \mathrm{~km}$. The intensity and magnitude averaged visual absolute magnitudes are $\left\langle M_{V}^{\text {int }}\right\rangle=0.74 \pm .05$ and $\left\langle M_{V}^{\mathrm{mag}}\right\rangle=0.78 \pm .05$ respectively.

Figures $4 b-d$ are plots of different physical quantities derived with $d=1.99 \times 10^{16} \mathrm{~km}$; the data were averaged for intervals $\Delta \varphi=0.01$. Note that the velocity $\dot{\vartheta}_{0}(\varphi) d$ shows a correlation with $p\left[v_{\gamma}-v_{\text {radial }}(\varphi)\right]$ only if it is averaged over a longer time. $\left(v_{\gamma}=-166.9 \mathrm{~km} \mathrm{~s}^{-1}\right.$, Liu \& Janes 1990) is the barycentric velocity, $p=1.32$ is the conversion factor. The phase difference between bolometric and visual maximum is $\Delta \varphi \approx 0.015$. 
Table 2. The averaged effective gravity, apparent acceleration of the continuum-forming layers, angular diameter, and acceleration for the phase interval $\varphi$ of standstills. The units are $\mathrm{m} / \mathrm{s}^{2}, \mathrm{~m} / \mathrm{s}^{2}, 10^{4} \mathrm{~km}, 10^{-10}$ radian, $10^{-19} \mathrm{radian} / \mathrm{s}^{2}$.

\begin{tabular}{lrrrrr}
\hline \hline$\varphi$ & $g_{\mathrm{e}}$ & $\ddot{R}_{\mathrm{a}}$ & $R_{\mathrm{a}}$ & $\vartheta_{0}$ & $\ddot{\vartheta}_{0}$ \\
\hline A: $.305-.315$ & 1.12 & $2.27:$ & 16.9 & 1.93 & $-4.75 \pm .56$ \\
B: $.545-.555$ & 1.58 & -0.25 & 11.3 & 1.88 & $-2.67 \pm .45$ \\
C: $.941-.954$ & 50.1 & 3.45 & 0.49 & 1.61 & $22.6 \pm 2.5$ \\
D: $.973-.983$ & 31.6 & 4.02 & 0.99 & 1.60 & $13.6 \pm 1.3$ \\
\hline
\end{tabular}

: Uncertain value.

Table 3. Distances and masses from Eqs. (17) and (18) without and with correction for $R_{\mathrm{a}}$, in units of pc and $\mathcal{M}_{\odot}$ respectively.

\begin{tabular}{rrrrr}
\hline \hline & \multicolumn{2}{c}{ I } & \multicolumn{2}{c}{ II } \\
& $d$ & $\mathcal{M}$ & $d$ & $\mathcal{M}$ \\
\hline $\mathrm{AC}$ & 535 & 0.68 & 555 & 0.85 \\
$\mathrm{AD}$ & 476 & 0.49 & 512 & 0.68 \\
$\mathrm{BC}$ & 593 & 0.57 & 647 & 0.66 \\
$\mathrm{BD}$ & 554 & 0.48 & 647 & 0.66 \\
$\mathrm{CD}$ & 665 & 0.31 & 647 & 0.66 \\
\hline
\end{tabular}

I: $R_{\mathrm{a}}=0, \ddot{R}_{\mathrm{a}}=0$.

II: $R_{\mathrm{a}}$ and $\ddot{R}_{\mathrm{a}}$ from Table $2, \vartheta_{\mathrm{a}}=R_{\mathrm{a}} / 647 \mathrm{pc}$.

\section{Discussion}

\subsection{Appraisal of the method, comparison with the BW technique}

The surface brightness method to determine stellar angular diameters is known in two variants. One of them (Barnes et al. 1978) uses the empirically determined surface brightness of non-variable stars and applies it to variables. The other variant is bound to static theoretical atmospheric models which are the sources of surface brightness and effective gravity. When applying them to variable stars a reliable surface brightness can be determined in the phases where a strong deviation from QSA is not expected. In the present paper the theoretical variant was used: the half angular diameter and the effective gravity were obtained from ATLAS atmospheric models as a function of phase. Concerning RR Lyrae stars arguments for the applicability of QSA were discussed in detail by Buonaura et al. (1985). Step 1 is identical in the present and BW methods. Step 2 is nearly the same in both methods, the difference is only that here the values $\vartheta_{0}$ from effective temperature and bolometric correction to $V$ are checked by a direct comparison of fluxes in $V$ and $R_{\mathrm{C}}$.

Steps 3 and 4 are principally different from the BW technique: all the data $\vartheta_{0}(\varphi), g_{\mathrm{e}}(\varphi)$ are used in a dynamical equation, i.e. in the $r$ component of the N-S equation averaged over the continuum-forming layers. Some terms could be neglected and the pressure-density stratification of atmospheric models with QSA was introduced. An essential assumption is that the function $g_{\mathrm{e}}=g_{\mathrm{e}}\left(C_{1}, \ldots\right)$ gives the uniform outward acceleration in $R_{1} \leq r \leq R_{2}$, and the sum $g_{\mathrm{e}}$ plus gravity determines the motion of the atmosphere at $R$. A term $g_{\mathrm{d}}$ was defined to account for the dynamical corrections to the stratification of QSA. In the range $T_{\mathrm{e}}, \lg g_{\mathrm{e}}$ of RR Lyrae stars the ATLAS models are convective. Its presumably small effect on the momentum balance of the pulsation could be incorporated in $g_{\mathrm{d}}$; however, it was neglected by the use of Eq. (12). In standstills of the atmosphere $g_{\mathrm{d}}$ is expected to be negligible and the averaged N-S equation is simplified to algebraic equations for the unknown quantities distance and mass. In the present method the mass plays an inherent role; it is determined simultaneously with the distance.

In the BW method the mass of a pulsating star plays an indirect role: in an equation of type Eq. (14) with $g_{\mathrm{d}} \equiv 0$ an assumed mass is introduced to derive $g_{\mathrm{e}}(\varphi)$ by differentiating the curve $-p v_{\text {radial }}(\varphi)$ numerically. This effective gravity is then used to select the atmospheric models from which the effective temperature is determined by a relation of type $T_{\mathrm{e}}\left(C_{i}\right)$, and the variation of angular diameter is determined. The kinematic equation

$$
\begin{aligned}
{\left[\vartheta_{0}\left(\varphi_{2}\right)-\vartheta_{0}\left(\varphi_{1}\right)\right] d } & =R_{0}\left(\varphi_{2}\right)-R_{0}\left(\varphi_{1}\right) \\
& =\int_{\varphi_{1}}^{\varphi_{2}} p\left[v_{\gamma}-v_{\text {radial }}(\varphi)\right] P \mathrm{~d} \varphi
\end{aligned}
$$

is solved for $d$ by various techniques (e.g. Liu \& Janes 1990; Cohen 1992; Jones et al. 1992), in the interval $\left[\varphi_{1}, \varphi_{2}\right]$ the atmospheres must be free of shocks. The primary error sources are $v_{\gamma}$ and $p$; it is obvious that their uncertainty can result in a considerable error in $d$ (Sabbey et al. 1995; Fernley 1994). The undulations reported in the present paper have a negligible effect on $d$ if $\left|\varphi_{2}-\varphi_{1}\right|$ is sufficiently large. Another small correction comes from the fact that $R(\varphi)$ would be more appropriate than $R_{0}(\varphi)$ if the radius change is determined by using radial velocities from CORAVEL (e.g. Liu \& Janes 1989) or from weak metallic lines (e.g. Oke et al. 1962).

In the present method $v_{\text {radial }}(\varphi), v_{\gamma}, p$, and an eventual arbitrary phase shift of the radius change from photometry and radial velocity (e.g. $\Delta \varphi=0.02$ for SU Dra, Siegel 1982) do not enter at all, the observation of radial velocities is not needed. However, a colour must observed, e.g. $U$ in Johnson system, which is a good indicator for gravity. In the BW method $g_{\mathrm{d}} \equiv 0$ can result in a negligible error, in the present method at selected phases $g_{\mathrm{d}}=0$, i.e. the unconditional use of Eq. (12), and the simplification of the velocity field to Eq. (13) with a single constant term can be assumed as a source of more uncertainty than in the BW method which involves the assumption $v_{r}(r, t)=\dot{R}(t)$ as well. Problems arising from the difference $S_{x}(\lambda)-S_{x}^{o}(\lambda) \not \equiv 0$ were discussed by Bessell (1990) where $S_{x}^{\mathrm{o}}(\lambda)$ represents the filter functions of an observatory. They are common both in the present method and in the BW method. This eventual systematic error is phase dependent.

\subsection{The results for SU Dra}

The distance derived in the present study agrees very well with its value $d=640 \mathrm{pc}$ from the BW analysis (Liu \& Janes 1990) if the subtle changes of $R_{\mathrm{a}}(\varphi)$ are taken into account. 
It is in accordance with the low absolute brightness of an RR Lyrae star if it is determined from a direct analysis. The higher absolute brightness of an RR Lyrae star from cluster properties is not bolstered by the present value of $d$. Concerning the short and long extragalactic distance scale (Gratton 1998) the discrepancy has been strengthened and an additional independent argument has been given for the short scale. If the change of $R_{\mathrm{a}}(\varphi)$ is neglected the discrepancy gets worse by $d=564 \pm 31$ pc. Furthermore, Eq. (19) has been verified: $p=1.32$ and $v_{\gamma}=-166.9 \mathrm{~km} \mathrm{~s}^{-1}$ (Liu \& Janes 1990) must essentially be correct since e.g. for $\varphi_{2}=0.8, \varphi_{1}=0.4$ an error $\Delta v_{\gamma}=1 \mathrm{~km} \mathrm{~s}^{-1}$ would result in an error

$\Delta d / d=\left[\left(\varphi_{2}-\varphi_{1}\right) p P \Delta v_{\gamma}\right] /\left[R\left(\varphi_{2}\right)-R\left(\varphi_{1}\right)\right] \approx 0.04$

i.e. roughly $-0.09 \mathrm{mag}$ in $M_{V}$. (It must be noted that Oke et al. 1962 argue for $v_{\gamma}=-161 \mathrm{~km} \mathrm{~s}^{-1}$ ). In the BW method the secondary error sources undulation and use of $R$ for $R_{0}$ result in $|\Delta d / d|<0.005$.

Liu \& Janes (1990) assumed $\mathcal{M}=0.55-0.65$ to get $\lg g_{\mathrm{e}}$ for the interval $0.4 \leq \varphi \leq 0.8$; they remarked that their $d$ was insensitive to the actual value $\left(0.6 \mathcal{M}_{\odot}\right)$. The basic pulsation equation of van Albada \& Baker (1971) gives $\mathcal{M}_{\mathrm{SU} \text { Dra }}=0.47 \mathcal{M}_{\odot}$, from which the present value $0.66 \pm .03 \mathcal{M}_{\odot}$ differs significantly, however, its acceptance must be supported by a smaller uncertainty in input physics.

The observed $-p v_{\text {radial }}(\varphi)$ (Liu \& Janes 1989; Oke et al. 1962) does not show the undulation of $\dot{\vartheta}_{0} d$, and the assumption of an eventually phase dependent conversion factor (Sabbey et al. 1995) cannot bring the curves to congruence. For a few variables in M5 and M92 a similar undulation of $\vartheta_{0}(\varphi)$ was shown graphically by Cohen (1992); however, it was not discussed. On a speculation level the most probable cause of this inconsistency is the non-negligible velocity gradient coupled with undulation of $\vartheta_{0}$. The radial velocities (especially those from CORAVEL) reflect the averaged velocity from the deep layers $\tau_{\text {Ross }} \approx 0.335$ while $\dot{\vartheta}_{0} d$ is the averaged velocity over the layers $0 \approx \tau<\tau_{\text {Ross }}=0.038$. At phase $\varphi \approx 0.75$ a weak hump is discernible on the CORAVEL curve which is correlated with the local maximum $\vartheta_{0}(0.78)$. It is, however, puzzling why there is such a loose correlation of $\dot{\vartheta}_{0}(\varphi) d$ with $p\left[v_{\gamma}-v_{\text {radial }}(\varphi)\right]$.

\section{Conclusions}

In the frame of quasi-static approximation for the atmosphere a new method has been described to determine distance and mass of pulsating stars. In elaborating the photometry there have been minor improvements compared to the conventional way in the BW analysis: the use of bolometric magnitudes from $T_{\mathrm{e}}$ minus the bolometric correction was supplemented with a comparison of physical fluxes from models and observations in a single filter band. It was postulated that at the phases of standstill the static ATLAS models give the effective outward acceleration in the atmosphere correctly. The effective gravities, the half angular diameters and their second derivatives with respect to time were introduced in the $r$ component of the NavierStokes equation from which we found distance and mass while neglecting dynamical corrections in the standstills of the atmosphere and making a small correction for the apparent radius changes. The method presented is purely photometric; radial velocity observations and their sophisticated interpretation are not needed.

As an example the light and colour curves of SU Dra have been interpreted. The agreement of the photometric angular diameters of different origin was within the expected error of a few percent. The distance we found is in excellent agreement with that from the BW analysis. A higher mass was found than that from pulsation equations; however, it is well reconcilable with the general mass values of RR Lyrae stars. An undulation of angular diameter has been found which can originate from a surface wave, restricted to the outermost parts of the atmosphere. It is synchronized with the main pulsation.

The results are encouraging for an application to distant RR Lyrae stars, e.g. for members of globular clusters or nearby galaxies. The necessary photometric observations are simple and can be done for large samples of these faint stars by moderate efforts. Distance derivations based on the BW method are available for some 30 field and a few cluster RR Lyrae variables (Gratton et al. 1997). These limited numbers could easily be increased by an order of magnitude if the present method were applied.

Acknowledgements. The author is grateful to J. M. Benkő, Z. Kolláth, L. Szabados for comments on the manuscript, to Fiorella Castelli for communicating her unpublished colours of Vega computed with the revised filters, and to J. Katgert for improving English presentation.

\section{Appendix A: The zero point of the magnitudes $\operatorname{UBV}(\mathbf{R I})_{C}$ of the Kurucz tables}

Tüg et al. (1977) measured the physical flux $\mathcal{I}_{\lambda}^{\text {(Vega) }}$ of Vega at 90 wavelength in the interval $3295 \AA \leq \lambda \leq 9090 \AA$ (Flagstaff flux calibration for $\alpha \mathrm{Lyr}$ ); this is $\mathcal{I}_{\lambda}$ for Eq. (7) in units $\operatorname{ergs~s}{ }^{-1} \mathrm{~cm}^{-2} \AA^{-1}$. The theoretical Eddington flux of Kurucz (1997, file fvega.pck belonging to $T_{\mathrm{e}}=9550 \mathrm{~K}, \lg g=3.95$, [metallicity] $=-0.5$ dex, $v_{\text {turb }}=2 \mathrm{~km} \mathrm{~s}^{-1}$ ) was multiplied by $4 \pi$, converted to the same units and interpolated to the 90 wavelengths: this is $\mathcal{F}_{\lambda}^{\text {(Vega) }}$ for Eq. (7). $A_{\lambda}$ was set to $=0$, $\vartheta_{0}^{\text {(Vega) }}=(7.95 \pm .02) \times 10^{-9}$ was found which agrees well with the interferometric value $(7.85 \pm .17) \times 10^{-9}$ (Hanbury Brown et al. 1974). Having $B C_{V}=-0.310, T_{\mathrm{e}}=9550 \mathrm{~K}$ from the theoretical model and $V=0.03$ mag from the observations the constant 0.636 is obtained in Eq. (8). (The parameters differ slightly from the observational values of Code et al. (1976): $B C_{V}=-0.250, T_{\mathrm{e}}=9660 \mathrm{~K}$.)

The observed flux $I_{\lambda}^{\text {(Vega) }}$ and the theoretical flux $\vartheta_{0}^{(\text {Vega) }} \mathcal{F}_{\lambda}^{\text {(Vega) }}$ were integrated with the filter functions for the system $U B V(R I)_{\mathrm{C}}$ (Bessell 1990) normalized to unity to obtain the physical fluxes belonging to the magnitudes of Vega. Table A.1 shows the results. $\mathcal{I}_{U}$ could not be computed from the Tüg et al. (1977) calibration because of a lack of data in the ultraviolet. The difference of $\mathcal{I}_{X}^{\text {(Vega) }}$ and $\vartheta_{0}^{\text {(Vega) }} \mathcal{F}_{X}^{\text {(Vega) }}$ originates from lines: $\mathcal{I}_{\lambda}^{\text {(Vega) }}$ was not measured at the strong lines and Balmer jump; it was interpolated linearly. 
Table A.1. Magnitudes and physical fluxes of Vega in units $10^{-8} \mathrm{ergs} \mathrm{s}^{-1} \mathrm{~cm}^{-2}$.

\begin{tabular}{lrrrrr}
\hline \hline$X$ & $U$ & $B$ & $V$ & $R_{\mathrm{C}}$ & $I_{\mathrm{C}}$ \\
\hline magnitudes & 0.022 & 0.027 & 0.03 & 0.039 & 0.035 \\
$\mathcal{I}_{X}^{\text {(Vega) }}$ & - & 32.9 & 18.1 & 10.5 & 5.95 \\
$\vartheta_{0}^{\text {(Vega) }} \mathcal{F}_{X}^{(\text {Vega) }}$ & 21.6 & 31.7 & 18.2 & 10.4 & 5.87 \\
\hline
\end{tabular}

For computing the observed flux of a star at zero air mass the lower row of Table A.1 must be used, an apparent magnitude $X$ is equivalent to a physical flux

$\mathcal{I}_{X}=10^{\left(X_{\text {Vega }}-X\right) / 2.5} \vartheta_{0}^{(\text {Vega })} \mathcal{F}_{X}^{(\text {Vega) }} \operatorname{ergs~s}^{-1} \mathrm{~cm}^{-2}$.

The monochromatic Eddington flux $H_{\lambda}$ of Vega (Kurucz 1997, fvega.pck) was integrated with the same normalized filter functions $U B V(R I)_{\mathrm{C}}$ of Bessell (1990); $-2.5 \lg \mathcal{H}_{X}=-23.835$, $-24.250,-23.645,-23.083,-22.370$ were found for $X=$ $U, B, V, R_{\mathrm{C}}, I_{\mathrm{C}}$ respectively. On the other hand the tabular Vega surface magnitudes are $X_{K}=-19.339,-19.755,-19.151$, $-18.590,-17.875$ if the improved filter functions of Bessell (1990) are used (Castelli 2002). The difference in these magnitudes is -4.495 i.e. the physical flux of the model is

$\mathcal{F}_{X}=10^{-\left(X_{K}-4.495\right) / 2.5} \mathrm{ergs} \mathrm{s}^{-1} \mathrm{~cm}^{-2}$

where $X_{K}$ is the tabulated magnitude $U, B, V, R_{\mathrm{C}}, I_{\mathrm{C}}$ (Kurucz 1997).

\section{References}

Albada van T. S., \& Baker, N. 1971, ApJ, 169, 311

Barcza, S. 2002, A\&A, 384, 460, Paper I

Barnes, T. G., Evans, D. S., \& Parsons, S. B. 1976, MNRAS, 174, 503

Baschek, B., Scholz, M., \& Wehrse, R. 1992, A\&A, 246, 374

Bessell, M. S. 1990, PASP, 102, 1181

Buonaura, B., Caccin, B., Onnembo, A., Russo, G., \& Sollazzo, C. 1985, Mem. Soc, Astron. Ital., 56, 153

Castelli, F. 1999, A\&A, 346, 564

Castelli, F. 2002, private communication

Code, A. D., Davis, J., Bless, R. C., \& Hanbury Brown, R. 1976, ApJ, 203, 417

Cohen, J. G. 1992, ApJ, 400, 528

Fernley, J. 1994, A\&A, 284, L16

Gautschy, A. 1987, Vistas Astron., 30, 197

Gratton, R. G., Pecci, F. F., Carretta, E., et al. 1997, ApJ, 491, 749

Gratton, R. G. 1998, MNRAS, 296, 739

Hanbury Brown, R., Davis, J., \& Allen, L. R. 1974, MNRAS, 167, 121

Jones, R. V., Carney, B. W., Strom, J., \& Latham, D. W. 1992, ApJ, 386, 646

Kurucz, R. L. 1979, ApJS, 40, 1

Kurucz, R. L. 1997, http: //cfaku5 . harvard.edu

Landau, L. D., \& Lifshitz, E. M. 1980, Hidrodinamika, Tankönyvkiadó, Budapest, vol. 6

Liu, T., \& Janes, K. A. 1989, ApJS, 69, 593

Liu, T., \& Janes, K. A. 1990, ApJ, 354, 273

Oke, J. B., Giver, L. P., \& Searle, L. 1962, ApJ, 136, 393

Sabbey, C. N., Sasselov, D. D., Fieldus, M. S., et al. 1995, ApJ, 446, 250

Siegel, M. J. 1982, PASP, 94, 122

Traving, G., Baschek, B., \& Holweger, H. 1966, Abhandlungen aus der Hamburger Sternwarte, VIII, No. 1

Tüg, H., White, N. M., \& Lockwood, G. W. 1977, A\&A, 61, 679 